The Einstein Telescope: a third-generation gravitational wave observatory

This article has been downloaded from IOPscience. Please scroll down to see the full text article.

2010 Class. Quantum Grav. 27194002

(http://iopscience.iop.org/0264-9381/27/19/194002)

View the table of contents for this issue, or go to the journal homepage for more

Download details:

IP Address: 194.94.224.254

The article was downloaded on 18/02/2013 at 07:48

Please note that terms and conditions apply. 


\title{
The Einstein Telescope: a third-generation gravitational wave observatory
}

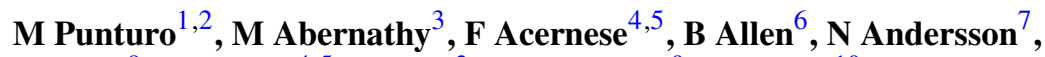

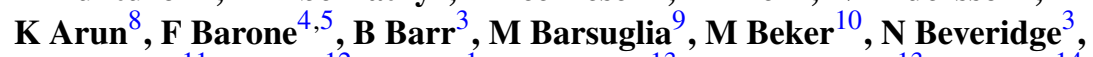

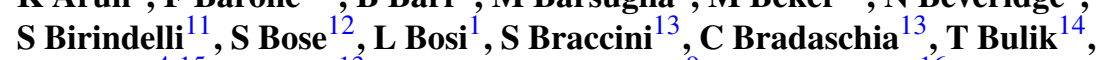 \\ E Calloni $^{4,15}$, G Cella $^{13}$, E Chassande Mottin ${ }^{9}, \mathbf{S ~ C h e l k o w s k i ~}^{16}$, \\ A Chincarini $^{17}, \mathbf{J ~ C l a r k}^{18}, \mathbf{E} \mathbf{C o c c i a}^{19,20}, \mathbf{C ~ C o l a c i n o ~}^{13}, \mathbf{J ~ C o l a s ~}^{2}$, \\ A Cumming $^{3}$, L Cunningham ${ }^{3}$, E Cuoco ${ }^{2}$, S Danilishin $^{21}$, K Danzmann ${ }^{6}$,

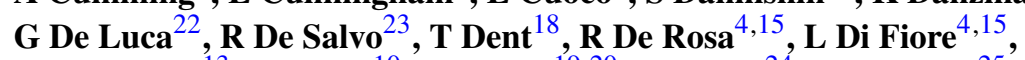 \\ A Di Virgilio ${ }^{13}$, M Doets ${ }^{10}$, V Fafone ${ }^{19,20}$, P Falferi $^{24}$, R Flaminio ${ }^{25}$, \\ J Franc $^{25}$, F Frasconi ${ }^{13}$, A Freise ${ }^{16}$, P Fulda ${ }^{16}$, J Gair ${ }^{26}$, G Gemme ${ }^{17}$, \\ A Gennai $^{16}$, A Giazotto $^{2,13}$, K Glampedakis ${ }^{27}$, M Granata ${ }^{9}$, H Grote ${ }^{6}$,

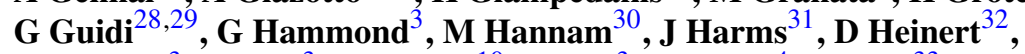 \\ M Hendry ${ }^{3}$, I Heng ${ }^{3}$, E Hennes $^{10}, \mathbf{S ~ H i l d ~}^{3}$, J Hough $^{4}, \mathbf{S ~ H u s a ~}^{33}$, \\ S Huttner $^{3}$, G Jones ${ }^{18}$, F Khalili ${ }^{21}$, K Kokeyama ${ }^{16}$, K Kokkotas ${ }^{27}$,

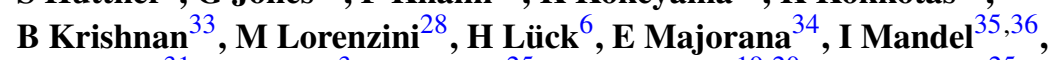

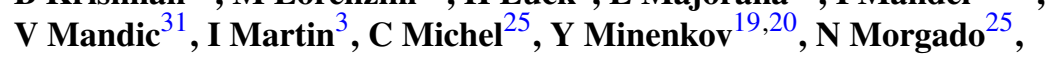 \\ S Mosca ${ }^{4,15}$, B Mours ${ }^{37}$, H Müller-Ebhardt ${ }^{6}$, P Murray $^{3}, \mathbf{R}$ Nawrodt $^{3}$,

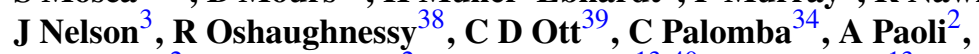 \\ G Parguez $^{2}$, A Pasqualetti ${ }^{2}$, R Passaquieti ${ }^{13,40}$, D Passuello ${ }^{13}$, \\ L Pinard $^{25}$, R Poggiani ${ }^{13,40}$, P Popolizio ${ }^{2}$, M Prato ${ }^{17}$, P Puppo $^{34}$, \\ D Rabeling ${ }^{10}$, P Rapagnani ${ }^{34,41}$, J Read ${ }^{33}$, T Regimbau ${ }^{11}$, H Rehbein ${ }^{6}$, \\ S Reid ${ }^{3}$, L Rezzolla ${ }^{33}$, F Ricci ${ }^{34,41}$, F Richard ${ }^{2}$, A Rocchi ${ }^{19}$, S Rowan ${ }^{3}$, \\ A Rüdiger $^{6}$, B Sassolas $^{25}$, B Sathyaprakash $^{18}$, R Schnabel $^{6}$,

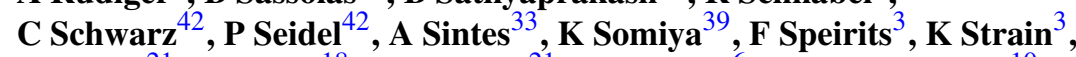

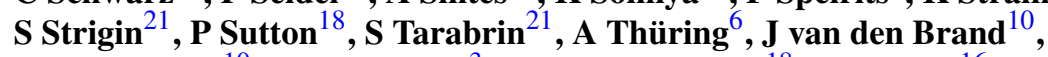 \\ $\mathrm{C}$ van Leewen ${ }^{10}, \mathbf{M}$ van Veggel $^{3}, \mathbf{C}$ van den Broeck ${ }^{18}, \mathbf{A}$ Vecchio $^{16}$,

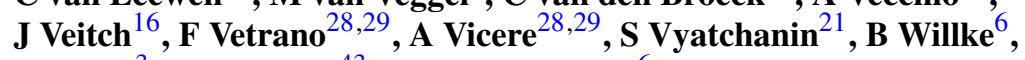

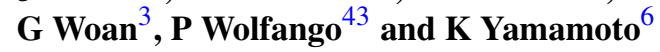 \\ ${ }^{1}$ INFN, Sezione di Perugia, I-6123 Perugia, Italy \\ 2 European Gravitational Observatory (EGO), I-56021 Cascina (Pi), Italy \\ ${ }^{3}$ Department of Physics and Astronomy, The University of Glasgow, Glasgow, G12 8QQ, UK \\ ${ }^{4}$ INFN, Sezione di Napoli, Italy \\ ${ }^{5}$ Università di Salerno, Fisciano, I-84084 Salerno, Italy \\ ${ }^{6}$ Max-Planck-Institut für Gravitationsphysik, D-30167 Hannover, Germany \\ ${ }^{7}$ University of Southampton, Southampton SO17 1BJ, UK \\ ${ }^{8}$ LAL, Université Paris-Sud, IN2P3/CNRS, F-91898 Orsay, France \\ 9 AstroParticule et Cosmologie (APC), CNRS; Observatoire de Paris-Université Denis \\ Diderot-Paris VII, France \\ ${ }^{10}$ VU University Amsterdam, De Boelelaan 1081, 1081 HV, Amsterdam, The Netherlands \\ ${ }^{11}$ Université Nice 'Sophia-Antipolis', CNRS, Observatoire de la Côte d'Azur, F-06304 Nice, \\ France \\ 12 Washington State University, Pullman, WA 99164, USA \\ 13 INFN, Sezione di Pisa, Italy
}


${ }^{14}$ Astro. Obs. Warsaw Univ. 00-478; CAMK-PAM 00-716 Warsaw; Bialystok Univ. 15-424; IPJ 05-400 Swierk-Otwock; Inst. of Astronomy 65-265 Zielona Gora, Poland

15 Università di Napoli 'Federico II', Complesso Universitario di Monte S. Angelo, I-80126

Napoli, Italy

16 University of Birmingham, Birmingham, B15 2TT, UK

17 INFN, Sezione di Genova, I-16146 Genova, Italy

18 Cardiff University, Cardiff, CF24 3AA, UK

19 INFN, Sezione di Roma Tor Vergata I-00133 Roma, Italy

${ }^{20}$ Università di Roma Tor Vergata, I-00133, Roma, Italy

${ }^{21}$ Moscow State University, Moscow, 119992, Russia

22 INFN, Laboratori Nazionali del Gran Sasso, Assergi l'Aquila, Italy

${ }^{23}$ LIGO, California Institute of Technology, Pasadena, CA 91125, USA

${ }^{24}$ INFN, Gruppo Collegato di Trento, Sezione di Padova; Istituto di Fotonica e Nanotecnologie, CNR-Fondazione Bruno Kessler, 38123 Povo, Trento, Italy

${ }^{25}$ Laboratoire des Matériaux Avancés (LMA), IN2P3/CNRS, F-69622 Villeurbanne, Lyon, France

${ }^{26}$ University of Cambridge, Madingley Road, Cambridge, CB3 OHA, UK

27 Theoretical Astrophysics (TAT) Eberhard-Karls-Universität Tübingen, Auf der Morgenstelle 10, D-72076 Tübingen, Germany

${ }^{28}$ INFN, Sezione di Firenze, I-50019 Sesto Fiorentino, Italy

${ }^{29}$ Università degli Studi di Urbino 'Carlo Bo', I-61029 Urbino, Italy

${ }^{30}$ Department of Physics, University of Vienna, Boltzmanngasse 5, A-1090 Vienna, Austria

31 University of Minnesota, Minneapolis, MN 55455, USA

32 Friedrich-Schiller-Universität Jena PF 07737 Jena, Germany

${ }^{33}$ Max Planck Institute for Gravitational Physics (Albert Einstein Institute) Am Mühlenberg 1, D-14476 Potsdam, Germany

34 INFN, Sezione di Roma 1, I-00185 Roma, Italy

35 Department of Physics and Astronomy, Northwestern University, Evanston, IL 60208, USA

${ }^{36}$ NSF Astronomy and Astrophysics Postdoctoral Fellow

${ }^{37}$ LAPP-IN2P3/CNRS, Université de Savoie, F-74941 Annecy-le-Vieux, France

38 The Pennsylvania State University, University Park, PA 16802, USA

${ }^{39}$ Caltech-CaRT, Pasadena, CA 91125, USA

${ }^{40}$ Università di Pisa, I-56127 Pisa, Italy

41 Università 'La Sapienza', I-00185 Roma, Italy

42 INFN, Sezione di Roma Tre and Università di Roma Tre-Dipartimento di Fisica, I-00146

Roma, Italy

${ }^{43}$ Università degli Studi di Firenze, I-50121, Firenze, Italy

E-mail: michele.punturo@pg.infn.it

Received 19 May 2010, in final form 22 June 2010

Published 21 September 2010

Online at stacks.iop.org/CQG/27/194002

\begin{abstract}
Advanced gravitational wave interferometers, currently under realization, will soon permit the detection of gravitational waves from astronomical sources. To open the era of precision gravitational wave astronomy, a further substantial improvement in sensitivity is required. The future space-based Laser Interferometer Space Antenna and the third-generation ground-based observatory Einstein Telescope (ET) promise to achieve the required sensitivity improvements in frequency ranges. The vastly improved sensitivity of the third generation of gravitational wave observatories could permit detailed measurements of the sources' physical parameters and could complement, in a multi-messenger approach, the observation of signals emitted by cosmological sources obtained through other kinds of telescopes. This paper describes the progress of the ET project which is currently in its design study phase.
\end{abstract}


PACS number: $40.80 . \mathrm{Nn}$

(Some figures in this article are in colour only in the electronic version)

\section{Introduction}

Interferometric gravitational wave $(\mathrm{GW})$ detectors have demonstrated the validity of their working principle by coming close to, or even exceeding, the design sensitivity of the initial instruments: LIGO [1], Virgo [2], GEO600 [3] and TAMA [4]. In the same infrastructures, currently hosting the initial GW detectors (and their limited upgrades, called 'enhanced' interferometers: eLIGO and Virgo+) a second generation of interferometers (so-called advanced detectors: 'Advanced LIGO' [5], 'Advanced Virgo' [6] and GEO-HF [3]) will be implemented over the next few years. The Laser Interferometer Space Antenna (LISA), a joint ESA-NASA mission expected to fly around 2020, is a space-borne detector to observe in the frequency range of $0.1 \mathrm{mHz}-0.1 \mathrm{~Hz}$ - a frequency range that is not accessible from ground. These detectors, based on technologies currently available, and partly already tested in reduced-scale prototypes, but still to be implemented in full scale, will show a sensitivity improved roughly by a factor of 10 with respect to the initial interferometers. Hence, a detection rate about a factor of 1000 larger than with the initial interferometers is expected, strongly enhancing the probability of detecting the signals generated by astro-physical sources. In particular, considering the predicted detection rate of the GW signal generated by a binary system of coalescing neutron stars [7], the sensitivity of the advanced interferometers is expected to guarantee the detection within months to a year at most.

Apart from extremely rare events, the signal-to-noise ratio (SNR) of detections in the 'advanced' detectors is likely to be still too low for precise astronomical studies of the GW sources and for complementing optical and x-ray observations in the study of fundamental systems and processes in the Universe. This consideration led the GW community to investigate the possibility of building a new (third) generation of detectors, permitting both to observe, with huge SNR, GW sources at distances similar to those detectable in the advanced detectors and to reveal GW signals at distances comparable with the sight distance of electromagnetic telescopes. As LISA will do for super-massive black holes $\left(M \simeq 10^{6} M_{\text {Sun }}\right.$ ), the Einstein Telescope (ET), thanks to this capability to inspect the GW signal in great detail, could herald a new era of routine GW astronomy for lighter astrophysical bodies.

To realize a third-generation GW observatory, with a significantly enhanced sensitivity (considering a target of a factor of 10 improvement over advanced detectors in a wide frequency range), several limitations of the technologies adopted in the advanced interferometers must be overcome and new solutions must be developed to reduce the fundamental and technical noises that will limit the next-generation detectors. But, mainly, new research facilities hosting the third-generation GW observatory apparatuses must be realized, to circumvent the limitations imposed by the current facilities. Hereafter, we will describe some of the possible scientific goals and some of the challenges of a third-generation GW observatory, as evaluated within the framework of the ET design study [8].

\section{ET science reach}

In figure 1 we plot a possible sensitivity curve of a third-generation GW detector [9]. This is by no means the final design goal but it sets the stage for studying what science questions can 


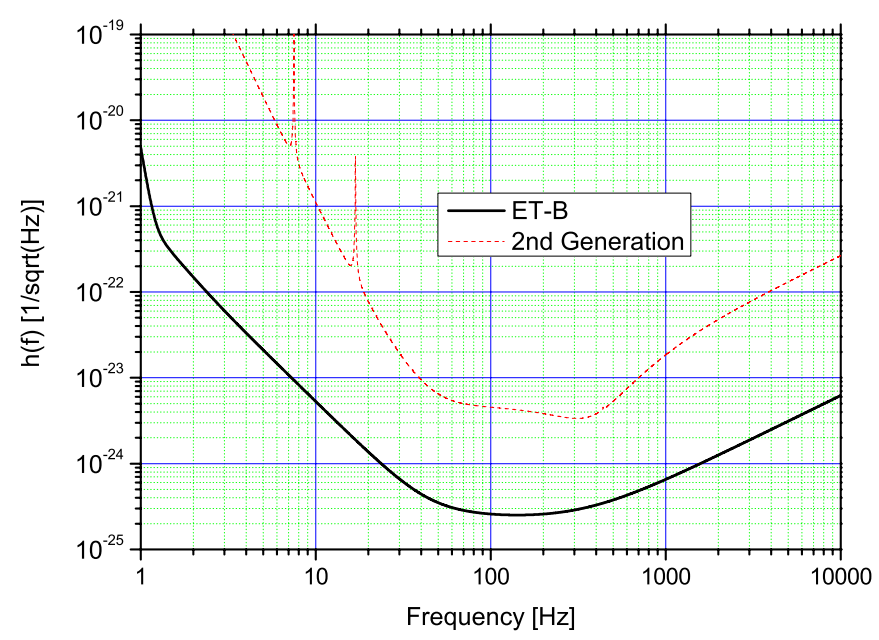

Figure 1. A possible sensitivity (solid curve) of an underground, long suspension, cryogenic, signal and power recycled single third-generation GW observatory (see table 1 in [9]) compared with a typical sensitivity curve of an advanced detector (dashed curve). It is worth underlining that the evaluation of the possible noise level of a third-generation GW observatory is an ongoing activity, still far from being concluded within the ET design study. For this reason the curves are updated regularly and labelled with progressive letters to be distinguished. In the solid curve (so-called ET-B), corresponding to a single wide-band detector, the suspension thermal noise contribution is not yet included.

be addressed with a third-generation detector. A detector with a sensitivity a factor 10 better than an advanced detector will open a new avenue for understanding the physical phenomena of extreme objects in the Universe. The study team has started putting together a vision document [10] detailing the scope of such a detector. Here we list a few examples of the science questions we can expect to pose with ET.

(i) Observation of compact binary coalescences would allow accurate measurement of the masses of neutron stars and masses and spins of black holes [11,12]. For instance, for binaries at a given distance, ET will measure masses and spins an order of magnitude better than advanced detectors. More importantly, it should be possible to determine the component masses of binaries to better than 5\% (except when the component objects are of comparable masses) over a wide range of masses from a few solar masses to hundreds of solar masses. From a population of such observations, it will be possible to infer the maximum mass of a neutron star (a long-standing open problem in theoretical physics) and constrain its equation of state [10]. The way this can be done is as follows: it is widely believed that short, hard gamma-ray bursts (shGRBs) are triggered by the coalescence of a compact binary in which one of the stars is a neutron star and the other a neutron star or a black hole. If this is the case, then one can reliably assume that the lighter of the components of a binary coalescence observed in coincidence is definitely a neutron star. A large enough sample should then give the mass function of neutron stars and tell us where the cutoff in the mass distribution is.

(ii) Advanced detectors should make the first coincident observations of binary mergers and shGRBs. One might not accumulate a sufficiently large population of such events with advanced detectors to fully understand the population of GRBs and their precursors. Advanced detectors could shed light on GRB progenitors (an outstanding problem 
in astronomy) and ET would allow classification of different types of GRBs, their demography, relationship to masses and spins of component stars, etc.

(iii) Simultaneous detection of neutrinos, electromagnetic and gravitational radiation from supernovae could help understand the mechanism behind type II supernovae and the astrophysics/physics of gravitational collapse. Furthermore, such observations also help to deduce or constrain the neutrino and graviton masses [13]. ET's range for supernovae is about $5 \mathrm{Mpc}$, within which one expects a supernova once every 2-5 years [14].

(iv) Comparing observations of massive binary star systems with numerical relativity predictions should allow testing general relativity and constrain alternative theories of gravity (such as the Brans-Dicke theory) [15].

(v) Inspiralling binary neutron stars (BNS) are ideal standard candles (or standard 'sirens'). A population of BNS merger events observed in coincidence with shGRBs can be used to measure cosmological parameters, GW observations helping to accurately estimate the luminosity distance and GRB hosts giving the source redshift [16-18].

(vi) ET will be sensitive to intermediate mass black hole binaries of total mass up to about a few thousand solar masses depending on the lower frequency cutoff [19-21]. The formation, abundance and coalescence rates of such systems are highly uncertain although it is plausible that intermediate mass black holes could be seeds of massive black holes that are now found at galactic nuclei, but they could also form in dense star clusters or by other means. If such systems exist, ET will provide an all sky survey of such objects up to redshifts of 2 or more.

(vii) Intermediate black holes, irrespective of when and where they form, could grow by accreting other compact objects such as stellar mass black holes and neutron stars. Here again the rates are unknown, but ET will be sensitive to the merger of stellar mass objects onto intermediate black holes at redshifts of $z=1$. Such events will be an invaluable tool to study the structure of spacetime geometry near massive black holes $[19,20]$, even though LISA's observation of stellar mass black holes inspiralling into supermassive black holes would be better at probing the spacetime geometry.

(viii) ET will be able to detect a stochastic background of GWs at the level of $\Omega_{\mathrm{GW}} \sim 10^{-12}$, where $\Omega_{\mathrm{GW}}$ is the energy density in stochastic background in units of the closure density of the Universe. This compares well with LISA's sensitivity of $\Omega_{\mathrm{GW}} \sim 10^{-11}$ in the frequency range of 2-20 mHz. Although ET's sensitivity is a few orders of magnitude poorer than that required to detect backgrounds predicted by inflationary Universe models, there is the possibility that phase transitions in the early Universe and other processes could give rise to a detectable background [10].

(ix) At the higher end of its frequency range, ET could observe normal modes in neutron stars excited in a host of astronomical events such as pulsar glitches, magnetar flares, soft-gamma repeaters, etc. GW, optical, x-ray, radio and gamma-ray windows would be invaluable tools for asteroseismology and the best way to probe neutron star interiors and to understand the equation of state of matter at extreme conditions of density, pressure, temperature and magnetic fields [10].

\section{Challenges for data analysis and the need for new search algorithms}

A detector with a sensitivity window and span as ET will pose new data analysis challenges. As in the case of LISA, there will be many classes of sources all visible at the same time, requiring a paradigm shift in the way data are currently being analysed. Some types of sources that can be assumed to be transients in current detectors will be in ET's band for many hours or even days. For such signals detector motion can no longer be neglected, requiring 


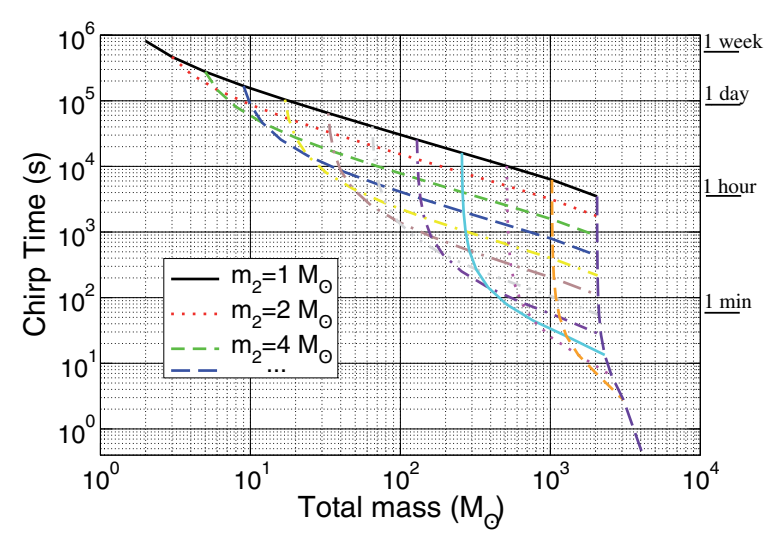

Figure 2. Duration of inspiral signals from binaries of total mass (as given by the $x$-axis) and mass $m_{2}$ of one of the component stars (as in the legend). Signals from BNS will last for about 9 days in the detector band. Many signals will last for more than a day but we will also have very short duration signals from intermediate mass black hole binaries. Curves are shown for the mass of one of the components varying from $1 M_{\odot}$ to $1024 M_{\odot}$, increasing by a factor of 2 at each step.

greater computational costs and the development of new search algorithms. A careful and comprehensive study of the data analysis challenges is currently underway. Here we discuss some of the basic problems a search algorithm should address in the ET era. Most of the following issues are relevant whatever the data analysis method followed, but more so in the case of a matched filtering search, e.g. for binary inspiral signals with a bank of templates.

As far as we can guess, compact binary mergers will dominate the ET observation band. Extrapolating the nominal rate of about one neutron star binary merger event per year within a distance of $100 \mathrm{Mpc}$ [7] to the distance reach of ET of about $20 \mathrm{Gpc}$, one expects to detect an event about every $6 \mathrm{~s}$. This extrapolation is, of course, not quite correct as it assumes sources to be uniformly distributed in space. In reality, we know that the star formation rate peaks at $z \sim 1$ and so the actual merger rate might be smaller by a moderate factor. Even so, merger signals from BNS and other compact binaries will be observed quite frequently in ET. We will, therefore, focus on the sort of problems this class of sources might pose.

(i) Figure 2 plots the duration of binary inspiral signals for systems with various masses, all starting at a frequency of $1 \mathrm{~Hz}$. Remarkably, inspiral signals could stay in the sensitive frequency band from as long as 10 days, for the lightest systems, to as briefly as only a few $100 \mathrm{~ms}$, for the heaviest ones.

(ii) The preponderance of signals in ET, as opposed to their rare occurrence in the 'advanced detectors', and their long duration means that signals will inevitably overlap with one another and that might cause confusion noise. It is necessary to evaluate the efficiency of the current algorithms in extracting overlapping signals buried in, say, Gaussian noise.

(iii) The occurrence of many overlapping signals could cause significant degradation of the parameter accuracies and thereby compromise ET's science potential. Moreover, the presence of many signals invalidates the assumption of stationarity of the data. How reliably can we extract signals and what are the parameter accuracies?

(iv) At $1 \mathrm{~Hz}$ the Doppler modulation due to Earth's rotation and revolution can be neglected for signals that last for considerably less than 1 day. For longer signals, Doppler modulations in signal amplitude and frequency become important. 
(v) The fact that a source's location in the sky is changing has to be taken into account in any analysis. Note, however, that at a frequency of $1 \mathrm{~Hz}$ the resolution of the detectors, even considering the baseline from the Earth's motion around the sun over 10 days, is only about 1 str and so this is not likely to be a big problem.

(vi) The biggest challenge might be matched filtering the data over the entire parameter space of binary systems to which the detector could be potentially sensitive. The number of templates grows roughly as $f_{s}^{-11 / 3}$ where $f_{s}$ is the frequency below which the detector accumulates negligible amount of signal-to-noise. The value of $f_{s}$ might be a factor 20-40 smaller in going from initial to third-generation detectors, thereby leading to a massive increase in the number of search templates and a corresponding increase in the trigger rate. Since the event rate is quite high (an event every 10 or $15 \mathrm{~s}$ ) it might be possible to use sub-optimal techniques to dig out most of the events, and these need to be explored.

(vii) Neutron stars and stellar mass black holes falling into intermediate mass black holes could last for several days in the band of sensitivity and will have close to millions of cycles. The complex orbits of such systems would pose a serious challenge to the analysis.

(viii) Amidst millions of binary inspiral signals we could have occasional burst signals from supernovae, neutron star quakes and the associated normal modes, continuous waves from spinning neutron stars, stochastic background of primordial or astrophysical origin, etc. How easy would it be to disentangle these interesting signals and characterize their properties?

The ET study team is working on a set of mock data challenges to test some of the questions posed above. These challenges are similar to the ones carried out in the context of LISA [22] and are open for anyone to participate. Our goal is to produce data sets of increasing complexity in order to provide an opportunity for us to address the data analysis and computational challenges posed by a third-generation detector.

\section{Technologies in ET}

To provide the ET with a sensitivity of a factor of 10 better than that of the 'advanced detectors', the relevant fundamental noise sources should be suppressed (neglecting the role of the so-called technical noises): the seismic and gravity gradient noise at very low frequency $(1-10 \mathrm{~Hz})$, the suspension thermal noise and quantum noise, related to the radiation pressure exerted on the suspended mirror by the photons in the main Fabry-Perot cavities (10-40 Hz), the thermal noise of the suspended mirrors (mainly the coating contribution, 40-200 Hz) and, finally, at higher frequencies, shot noise component of the quantum noise.

\subsection{Seismic and gravity gradient noise reduction}

The seismic noise affects the sensitivity at low frequency of the current GW interferometric detectors. In the Virgo detector, the so-called super attenuator (SA) [23] has shown its capability to filter the seismic noise below the expected thermal noise. The performances of the SA have been confirmed to be compliant with the attenuation requirements in Advanced Virgo [24] and, considering as reference a seismic noise linear spectral density of $5 \times 10^{-9} / f^{2} \mathrm{~m} \mathrm{~Hz}^{-1 / 2}$, value measured in the Kamioka (Japan) mine, selected for the construction of the LCGT interferometer [25], it is expected to be easily re-scalable to be compliant with the more restrictive ET noise requirements at low frequency [24]. 
The gravity gradient noise is due to the direct coupling of the suspended test-mass displacement with the mass vibration in the soil layers, perturbed by seismic waves, via the mutual attraction force expressed by Newton's universal law of gravitation [26-28]. Obviously the importance of this disturbance depends on the seismic noise level and on the contribution of the other low-frequency noise sources to the noise budget. In the third generation of GW detectors, the more stringent requirements in terms of sensitivity at low frequency enhance the importance of this noise source and enforce the need to realize the ET in an underground and quiet site.

\subsection{Thermal noise reduction}

Under the 'thermal noise' label are grouped all those processes that modulate the optical path of the light in the interferometer coupling it to the Brownian fluctuation or to the stochastic fluctuation of the temperature field in the optical components. Usually, one distinguishes between the suspension thermal noise, affecting the position of the test masses through the fluctuations of the suspension wires or fibres, and the mirror thermal noise, which is the sum of all the overlapping fluctuation and dissipation processes occurring in the test masses and in its high-reflectivity coatings. The strategies to reduce the thermal noise impact in the second-generation GW detectors are essentially an evolution of what has been applied in the initial detectors and are based on the reduction of the dissipation in the suspension system, in order to concentrate all the fluctuation energy into the normal modes of the system, resulting in a low noise level off-resonance.

In addition to these strategies, in ET we propose to act directly on the total fluctuation energy, by reducing the operative temperature of the suspended optics. Hence, cryogenics is one of the most appealing technologies to reduce the thermal noise of the optics suspension in the third generation of GW observatories. The design of the cryogenic suspension and of its cooling system is progressing in the ET design study and possible material candidates for the test masses and suspension fibres have been identified in sapphire (as already done [29, 30] for LCGT) and silicon [8, 31, 32].

\subsection{Quantum noise reduction}

Quantum noise in interferometric GW detectors can be understood as the coupling of vacuum fluctuations with the optical readout fields inside the interferometer. This coupling causes an uncertainty in the phase and amplitude of the probe beam, which affects the interferometer output signal in two ways. The phase uncertainty directly spoils the phase measurement of the Michelson interferometer; this effect is called shot noise. The amplitude uncertainty, or in other words, the changing amplitude of the light, will cause the light pressure on the test masses to change, which correspondingly causes motions of the test masses; this effect is called radiation pressure noise. Quantum noise is the sum of shot noise and radiation pressure noise and in the classical Michelson interferometers poses a fundamental limit to the sensitivity of the detector, the so-called standard quantum limit (SQL).

Techniques to improve the sensitivity beyond the SQL are called quantum noise reduction (QNR) or somewhat misleadingly quantum non-demolition (QND) schemes. A more detailed introduction to this topic is given in [8], see also [33] for a review of QND schemes discussed in the context of the ET. Currently we aim at using a Michelson interferometer with signal recycling and a squeezed light field injected into the interferometer output for reducing the quantum noise in the ET. This method would benefit greatly from a 'xylophone' approach (see section 4.4). Alternatively a Sagnac topology is studied as a possible alternative; the 


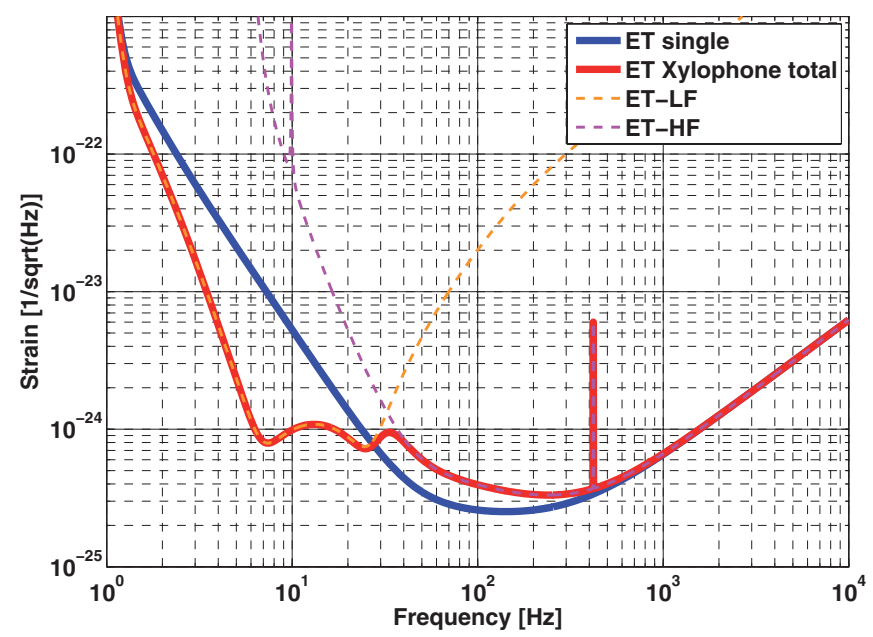

Figure 3. Sensitivity of a third-generation GW observatory implemented by two frequencyspecialized (LF and HF) detectors (xylophone topology [34], curve so-called ET-C), with respect to a single wide frequency range interferometer ET implementation [9].

Sagnac interferometer is insensitive to radiation pressure noise; so far, however, much less experimental expertise has been gained with this topology.

\subsection{Multiple interferometer detector}

As described in the previous subsections, to realize a third-generation GW detector, the technologies currently operative in the initial detectors and planned for the advanced detectors must be further advanced and new solutions must be adopted. The cross-compatibility between the different solutions becomes a crucial issue; for example, the requirements imposed by the reduction of the quantum noise conflicts with those imposed by the thermal noise suppression. This technological difficulty in realizing a single wide-band third-generation detector can be avoided. The base line currently favoured in the ET design study [34] is a combination of two interferometers, specialized on different frequency bands: the so-called xylophone philosophy [35]. Here the output of a low-frequency-specialized detector is combined with the output of a high-frequency machine. The former could be a cryogenic interferometer at an underground site, with long suspensions, but moderate optical power, whereas the highfrequency interferometer could essentially be a long arm advanced detector, implementing squeezed light states, a very high-power laser and large test masses. A possible realization of such a xylophone strategy, evaluated in [34] ('ET-C') for the ET design study, is plotted in figure 3 and compared with the single-interferometer implementation ('ET-B') of the ET observatory, described in [9].

\section{Site and infrastructure}

In subsection 4.1 it has been assumed that the required seismic noise spectral density, compliant with the ET sensitivities shown in figure 3, corresponds to the noise measured in an underground site. In effect, one of the major activities to be accomplished in the ET design study phase is the identification of the noise requirements of the site hosting the 
observatory and the compilation of a candidate list in Europe. The first results of this study indicate that the site hosting the ET observatory should be located a few hundred metres underground, in order to reduce the dominant disturbance of surface seismic waves, in a region with reduced anthropogenic activity, far from main natural noise sources such as the ocean. To reduce the gravity gradient noise a 'soft' soil is recommendable, but it causes major construction difficulties and additional costs.

Another important characteristic of the site is the length of the tunnels hosting the main cavities' arms. To gain a factor of 10 with respect to the advanced detectors, the length of the ET arms should be about $10 \mathrm{~km}$; as this length agrees well with the average lifetime of tunnel boring machines, which need to be bought, it also optimizes the costs in this respect. In effect, the cost of the site excavation and of the hosting infrastructure, under evaluation in the ET project, will surely dominate the overall budget of the project. For this reason it will be mandatory to maximize the usage of the site, for example, installing multiple detectors in the tunnels.

\subsection{Detector geometry}

All the currently active GW interferometric detectors are L-shaped, with orthogonal arms, since this geometry maximizes the sensitivity of a single detector with respect to the arm length. But other geometries are possible, like triangular-shaped detectors already proposed in the past [36], and could become preferable if the selection criteria are more complex than the simple sensitivity maximization. As analysed in detail in [37], a triangular-shaped observatory, composed of three co-located interferometric detectors, could present many advantages in terms of redundancy, signal reconstruction and cost/benefit minimization, and this geometry is becoming the baseline option of the ET project.

\section{ET project evolution}

The ET design study is supported for 3 years (2008-2011) within the European Community Seventh Framework Programme (FP7), having the aim of delivering the conceptual design of such a research infrastructure, investigating the technological feasibility, the science targets, the site requirements and preparing a costing draft for the infrastructure.

After this phase, preparatory activity is expected to be necessary to define the technological details, and the legal and organizational issues. The start of construction (2018-2019) is expected to occur after the first detection of GWs, which is reckoned to happen within at most 1 year after the advanced detectors have reached their nominal sensitivity. The construction and commissioning timeline of ET is under study, but about 7-8 years are expected to be necessary before we have the first data available.

\section{Acknowledgments}

The authors gratefully acknowledge the help of Albrecht Rüdiger in correcting this paper. This work has been performed with the support of the European Commission under the Framework Programme 7 (FP7) 'Capacities', project Einstein Telescope (ET) design study (Grant Agreement 211743), http://www.et-gw.eu/ 


\section{References}

[1] Abbott B P et al 2009 LIGO: the laser interferometer gravitational-wave observatory Rep. Prog. Phys. 72076901

[2] Accadia T, Swinkels B L and the VIRGO Collaboration 2010 Commissioning status of the Virgo interferometer Class. Quantum Grav. 27084002

[3] Grote H and the LIGO Scientific Collaboration 2010 The GEO 600 status Class. Quantum Grav. 27084003

[4] Arai K and the TAMA Collaboration 2008 Recent progress of TAMA300 J. Phys.: Conf. Ser. 120032010

[5] Harry G M and the LIGO Scientific Collaboration 2010 Advanced LIGO: the next generation of gravitational wave detectors Class. Quantum Grav. 27084006

[6] Acernese F et al Advanced Virgo baseline design VIR-027A-09 (https://tds.ego-gw.it/q1/?c=6589)

[7] Abadie $\mathrm{J}$ et al 2010 Predictions for the rates of compact binary coalescences observable by ground-based gravitational-wave detectors arXiv:1003.2480

[8] Punturo M et al 2010 The third generation of gravitational wave observatories and their science reach Class. Quantum Grav. 27084007

[9] Hild S, Chelkowski S and Freise A 2008 Pushing towards the ET sensitivity using 'conventional' technology arXiv:0810.0604v2 [gr-qc]

[10] Amaro-Seoane $\mathrm{P}$ et al 2009 Einstein Telescope design study: vision document https://workarea.et-gw.eu/et/wg4-astrophysics/visdoc/. ET internal note: ET-031-09

[11] Van Den Broeck C 2006 Binary black hole detection rates in inspiral gravitational wave searches Class. Quantum Grav. 23 L51-8

[12] Van Den Broeck C and Sengupta A S 2007 Phenomenology of amplitude corrected post-Newtonian gravitational waveforms for compact binary inspiral: I. Signal-to-noise ratios Class. Quantum Grav. 24 155-76

[13] Arnaud N et al 2002 Gravity wave and neutrino bursts from stellar collapse: a sensitive test of neutrino masses Phys. Rev. D 65033010

[14] Ando S, Beacom J F and Yüksel H 2005 Detection of neutrinos from supernovae in nearby galaxies Phys. Rev. Lett. 95171101

[15] Arun K G and Will C M 2009 Bounding the mass of the graviton with gravitational waves: effect of higher harmonics in gravitational waveform templates Class. Quantum Grav. 26155002

[16] Schutz B F 1986 Determining the Hubble constant from gravitational wave observations Nature 323 310-1

[17] Sathyaprakash B S, Schutz B and Van Den Broeck C 2009 Cosmography with the Einstein telescope arXiv:0906.4151v1 [astro-ph.CO]

[18] Nissanke S, Hughes S A, Holz D E, Dalal N and Sievers J L 2009 Exploring short gamma-ray bursts as gravitational-wave standard sirens arXiv:0904.1017

[19] Gair J R, Mandel I, Coleman Miller M and Volonteri M 2009 Exploring intermediate and massive black-hole binaries with the Einstein telescope arXiv:0907.5450

[20] Gair J R, Mandel I, Sesana A and Vecchio A 2009 Probing seed black holes using future gravitational-wave detectors Class. Quantum Grav. 26204009

[21] Amaro-Seoane P and Santamaria L 2009 Detection of IMBHs with ground-based gravitational wave observatories: a biography of a binary of black holes, from birth to death arXiv:0910.0254

[22] Babak S et al 2010 The mock LISA data challenges: from challenge 3 to challenge 4 Class. Quantum Grav. 27084009

[23] Braccini S et al 2005 Measurement of the seismic attenuation performance of the Virgo superattenuator Astropart. Phys. 23 557-65

[24] Acernese F et al 2010 Measurements of superattenuator seismic isolation by Virgo interferometer Astropart. Phys. 33 182-9

[25] Uchiyama T et al 2004 Present status of large-scale cryogenic gravitational wave telescope Class. Quantum Grav. $21 \mathrm{~S} 1161$

[26] Saulson P R 1984 Terrestrial gravitational noise on a gravitational wave antenna Phys. Rev. D $30732-736$

[27] Beccaria M et al 1998 Relevance of Newtonian seismic noise for the Virgo interferometer sensitivity Class. Quantum Grav. 15 3339-62

[28] Hughes S A and Thorne K S 1998 Seismic gravity-gradient noise in interferometric gravitational-wave detectors Phys. Rev. D 58122002

[29] Uchiyama T et al 2000 Mechanical quality factor of a sapphire fiber at cryogenic temperatures Phys. Lett. A 273 310-5

[30] Tomaru T et al 2002 Maximum heat transfer along a sapphire suspension fiber for a cryogenic interferometric gravitational wave detector Phys. Lett. A $301215-9$

[31] Alshourbagy M et al 2006 First characterization of silicon crystalline fibers produced with the $\mu$-pulling technique for future gravitational wave detectors Rev. Sci. Instrum. 77044502 
[32] Reid S et al 2006 Mechanical dissipation in silicon flexures Phys. Lett. A 351 205-11

[33] Müller-Ebhardt H et al 2009 Review of quantum non-demolition schemes for the Einstein Telescope ET-010-09

[34] Hild S, Chelkowski S, Freise A, Franc J, Morgado N, Flaminio R and DeSalvo R 2010 A xylophone configuration for a third-generation gravitational wave detector Class. Quantum Grav. 27015003

[35] Conforto G and DeSalvo R 2004 Proposal for lower frequency companions for the advanced LIGO gravitational wave interferometric detectors Nucl. Instrum. Methods Phys. Res. A 518 228-32

[36] Winkler W et al (eds) 1985 Plans for a large gravitational wave antenna in Germany MPQ Report 101

[37] Freise A, Chelkowski S, Hild S, Del Pozzo W, Perreca A and Vecchio A 2009 Triple Michelson interferometer for a third-generation gravitational wave detector Class. Quantum Grav. 26085012 